\title{
TIEMPO Y LUGAR DE LOS MOVIMIENTOS SOCIALES TRANSNACIONALES*
}

\author{
JUAN PABLO BOHÓRQUEZ MONTOYA"* \& TITO HERNANDO PÉREZ PÉREZ *** \\ UNIVERSIDAD DE LA SALLE
}

Recibido/ Received/ Recebido: 29/12/2011 - Aceptado/ Accepted/Aprovado: 20/04/2011

\begin{abstract}
Resumen
Los movimientos sociales transnacionales han surgido en un largo proceso histórico. Estos movimientos han creado un espacio social distinto, en el cual han establecido una serie de lazos simbólicos, formas de organización y mecanismos de articulación que les han permitido plantearse como sujetos de cambio social en un mundo cada vez más interconectado. No obstante, estos movimientos han entrado, supuestamente, en un proceso de languidecimiento. ¿Cómo han surgido y qué crean estos movimientos sociales? ¿Cuáles son las posibilidades de estos movimientos de continuar o plantearse a largo plazo como sujetos de transformación social en el espacio internacional? Son las dos preguntas que guían esta investigación. El documento termina reconociendo la importancia de estos movimientos en la denuncia y puesta evidencia de las contradicciones del capitalismo contemporáneo.
\end{abstract}

Palabras clave: Movimientos sociales transnacionales, espacio social transnacional, globalización, articulación social.

\section{TIME AND PLACE OF TRANSNATIONAL SOCIAL MOVEMENTS}

\begin{abstract}
Transnational social movements have emerged in a long historical process. These movements have created a distinct social space, in which, there is a series of symbolic strings, organization forms and articulation mechanisms which have made them to establish as subjects of social change in an each time more interconnected world. However, these movements have entered, supposedly, in a waning process. How emerge and create these social movements? Which are the possibilities of these movements to continue or establish long term as social transformation subjects in international space? These two questions guide this research. The paper ends recognizing the importance of these movements in denouncing and evidencing contemporary capitalism contradictions.

Keywords: Transnational social movements, transnational social space, globalization, social articulation.
\end{abstract}

Este trabajo de revisión es producto de la investigación sobre movimientos sociales colombianos agrarios de carácter transnacional que se viene desarrollando en Programa de Trabajo Social de la Universidad de la Salle, Bogotá.

* Economista, psicólogo, magíster en análisis de problemas políticos, económicos e internacionales contemporáneos. Candidato al doctorado en ciencia política de la Universidad Laval, Quebec (Quebec), Canadá. Áreas de especialización: sociología política, filosofía política y estudios internacionales. Docente de la Facultad de Ciencias Económicas y Sociales, Programa de Trabajo Social - Universidad de la Salle. Miembro del Centro de Investigación Hábitat, Desarrollo Paz (Cihdep), Grupo de Derechos Humanos, Cultura y Democracia, Universidad de la Salle. Correo electrónico: jubohorquez@unisalle.edu.co, jpablobm@gmail.com.

*** Lic. Ciencias Sociales, estudios en derecho y estudios de maestría en análisis de problemas políticos, económicos e internacionales contemporáneos. Magíster en Docencia. Docente de la Facultad de Ciencias Económicas y Sociales, Programa de Trabajo Social - Universidad de la Salle. Docente de la Maestría en Educación en la Universidad Santo Tomás de Aquino. Miembro del Centro de Investigación en Hábitat, Desarrollo y Paz (Cihdep), Grupo de Derechos Humanos, Cultura y Democracia, Universidad de la Salle. Correo electrónico: tperez@unisalle.edu.co 


\title{
TEMPO E LUGAR DOS MOVIMENTOS SOCIAIS TRANSNACIONAIS
}

\section{Resumo}

\begin{abstract}
Os movimentos sociais transnacionais são fruto de um processo histórico prolongado. Esses movimentos têm possibilitado a criação de espaços sociais diferentes em que surgem laços simbólicos, formas organizacionais e mecanismos de articulação que lhes permitem transformar-se em sujeitos de mudança social em um mundo cada vez mais interconectado. No entanto, supostamente, têm alcançado um estado de elanguescimento. Como surgem e que produzem esses movimentos sociais? Quais são as possibilidades desses movimentos continuarem e serem sujeitos de transformação social ao longo prazo no ambiente internacional? Estas duas perguntas guiam esta pesquisa. Ao final, o documento reconhece a importância desses movimentos na denuncia das contradições do capitalismo atual.
\end{abstract}

Palavras chave: Movimentos sociais transnacionais, espaço social transnacional, globalização, articulação social.

Bohórquez, J. \& Pérez, T. (2011). Tiempo y lugar de los movimientos sociales transnacionales. En: Revista de la Facultad de Ciencias Económicas de la Universidad Militar Nueva Granada. rev. fac.cienc.econ, XIX (1).

JEL: O19, O54, Z13.

\section{Introducción}

El auge de los movimientos sociales globales, altermundialistas, transnacionales o antiglobalización, ha entrado aparentemente en declive. La indagación de estos y su significado, nos obliga a pensar los términos con que se los caracteriza; por cuáles etapas han atravesado, especialmente desde la década del 60 del siglo pasado; qué tipo de relaciones establecen con la denominada globalización del capital; $y$, finalmente, cuál ha sido su contribución al agenciamiento colectivo. Este recorrido nos permitirá comprender cuáles son sus posibilidades reales en un entorno de crisis económica mundial del capital.

\section{Algunos elementos metodológicos}

Para responder a estas preguntas se ha desarrollado un proceso investigativo en dos fases. La primera, es la realización de un estado del arte sobre la cuestión, para lo cual se acude a las herramientas de la investigación documental y el análisis de contenido y cuyo primer resultado es este artículo. La segunda, que se encuentra en curso, es el levantamien- to de la información a través de la aplicación de entrevistas semi-estructuradas a diferentes líderes y lideresas de los movimientos campesinos tanto en Colombia como en el ámbito internacional.

El trabajo se fundamenta especialmente en el análisis documental y para su desarrollo se acudió a la propuesta metodológica de Pinto \& Gálvez (1996), quienes afirman que el análisis de contenido de los documentos se ha constituido en un análisis del discurso de los documentos. Se pasa entonces de la realización exclusiva de un análisis lingüístico a lectura sobre la producción, comprensión y producción de textos. En este mismo sentido, se retoman los aportes de Van Dijik (1996) quien señala que el análisis ideológico del análisis del lenguaje y del discurso, supone poner al "descubierto" la ideología de hablantes y escritores a través de lecturas minuciosas de los contenidos; se leen los discursos (textos) desde un perspectiva sociopolítica, y de esta forma se establecen las relaciones de los discursos con las estructuras sociales.

En síntesis, así se utilizan diferentes herramientas de la investigación cualitativa y la revisión documen- 
tal lo que implica la "triangulación metodológica", desde la cuales logra la completa, rica, detallada y densa elaboración de los fenómenos sociales, entre ellos, de los movimientos sociales (Snow \& Trom, 2002, 151), pudiéndose determinar su momento histórico, es decir, su trayectoria que implica sus condiciones de posibilidad espacio-temporales. Lo anterior bajo la premisa, como lo plantea Carlo Ginzburg (2003, 34), que las fuentes:

"ne sont ni des fenêtres ouvertes, comme le croient les positivistes, ni de murs qui obstruent la vue, comme le soutient les sceptiques : en fait, c'est à des vitres déformantes qu'il faut les comparer. L'analyse des distorsions spécifiques à chaque source implique déjà un élément constructif".

"[no son ni ventanas abiertas, como lo creen los positivistas, ni muros que obstruyen la vista, como lo sostienen los escépticos: de hecho, son cristales deformantes que es necesario comparar. El análisis de las distorsiones de cada fuente implica ya un elemento constructivo]".

\section{3. ¿Qué son los movimientos sociales globales o transnacionales?}

Los movimientos sociales globales, o transnacionales, son aquellos que construyen un espacio social al conectar distintos países o unidades nacionales, es decir, formaciones sociales. Este espacio social, denominado generalmente transnacional (Faist, 2000), se crea a través de lazos de orden simbólico y social producidos por la "unidad" de distintos movimientos sociales - del orden subnacional, regional o local-, de las relaciones de éstos con los Estados, las fuerzas sociales oponentes y las instituciones nacionales y multinacionales, entre otras.

El solo hecho de plantear la existencia de un espacio social transnacional obliga a abordar distintos problemas que se conjugan en la expresión "articulación". En otras palabras, conlleva establecer cuáles son las implicaciones de dicha imbricación, cuál es su efecto en las formaciones sociales que entran en contacto con otras agrupaciones, locales y externas, y cómo se acoplan entre sí los objetivos y luchas de movimientos sociales tan distintos entre sí, como por ejemplo de campesinos franceses y colombianos, en temas no comunes como lo son la reforma agraria y las distintas composiciones ideológicas y de clase.

Para algunos autores, un movimiento social transnacional se da, primero, cuando un movimiento social logra la exteriorización de alguno de sus problemas, el cual pasa a ser un tema de lucha y controversia común de otros movimientos sociales ajenos a la formación social de la cual ha surgido; segundo, cuando los movimientos disponen de aliados externos; tercero, cuando se logra construir un sentido común, y se identifican objetivos y adversarios compartidos por todos los movimientos que entran a formar parte de ese nuevo movimiento; cuarto, a través de la disposición de medios de comunicación rápidos y baratos; quinto, por la capacidad de combinar formas de acción colectiva para enfrentar los blancos escogidos u obligados (Borras, 2008). ${ }^{1}$ Los anteriores elementos permitirían a los movimientos sociales, que en su acción colectiva trascienden las fronteras nacionales, étnicas e incluso de clase (Bohórquez Montoya, 2009), -que permite trascender el lugar asignado a distintos grupos humanos en la producción material y cultural de la sociedad (Marx, 1985)-, convertirse en un movimiento que sobrepasa la sumatoria de sus componentes al crear nuevas espacios y lugares sociales donde desarrollar sus luchas y plantear sus reivindicaciones.

\section{Momentos históricos de los movimientossocialestransnacionales}

Los movimientos sociales transnacionales tienen sus antecedentes en los movimientos internacionalistas del siglo XIX y principios del XX, que vieron

1 Borras recoge los postulados de la escuela de la estructura de oportunidades políticas y los trabajos de las redes. (Véase especialmente: Khagram, Riker \& Sikkink, 2002; Tarrow, 2005). 
limitado y modificado su alcance como consecuencia del cierre de las fronteras, después de 1914, y la reconfiguración del orden internacional surgido después de la Segunda Guerra Mundial. No obstante, ya hacia finales de los años 60, en particular por obra de los cambios en la estructura migratoria internacional, el desarrollo de los medios de comunicación y de transporte, el fin de la guerra de Vietnam, la ruptura del sistema de Bretton Woods, la globalización y los principios de gobernanza global, se puso en evidencia la existencia de otros agentes sociales - distintos de los Estados y sus organizaciones intergubernamentales- que estaban presentes en el ámbito internacional, cual las organizaciones no gubernamentales, las corporaciones multinacionales, las asociaciones de todo orden, los partidos políticos, y diversos movimientos sociales.

La presencia de estos últimos se ha delimitado de acuerdo con el carácter y objetivos de sus luchas, entendidas como la oposición, resistencia, acciones y/o propuestas de un movimiento grupo, fracción o clase social para imponer sus intereses objetivos en relación con otros grupos sociales y el mismo Estado (Poulantzas, 1986, 172). Así, Jeffrey M. Ayres $(2005,13)$ propone la siguiente periodización: desde finales de los años 60 hasta principios de los 90, los movimientos sociales transnacionales, con distintas actividades y enfoques, se caracterizan por su afán de establecer conexiones entre los distintos problemas sociales, políticos y económicos y las instituciones que procuran el avance de las políticas neoliberales ${ }^{2}$. En este contexto surgen los movimientos de las mujeres en el desarrollo, las movilizaciones en contra de la deuda externa y los programas de ajuste de organismos como el Banco Mundial, entre otros. Aquí sería pertinente ubicar, también, el movimiento pacifista que nunca desaparecería, y los movimientos de trabajadores, campesinos y ambientalistas (divididos entre el Sur y el Norte). En este período, dado su carácter de protesta contra lo global, los movimientos reciben en consecuencia el apelativo de movimientos an- tiglobalización y entre ellos cabe destacar el de los Zapatistas y el así llamado Jubileo 2000.

Un segundo período abarca la década de los años noventa, cuyo símbolo es la denominada "Batalla de Seattle" (29 de noviembre al 3 de diciembre de 1999). Este lapso está dominado por movimientos más propositivos, con agendas altamente estructuradas y propuestas de alternativas, tanto al neoliberalismo como a la globalización del capital, en las que se cuestionan las credenciales democráticas y la legitimidad de las instituciones multilaterales como el Banco Mundial, el Fondo Monetario Internacional y la Organización Mundial del Comercio, entre otras. En este período se enmarca asimismo la consolidación de la Vía Campesina, de movimientos como Nuestro Mundo No Es para la Venta, Mujeres y Desarrollo Alternativo y el surgimiento del Foro Social Mundial.

Para algunos autores, dicho lapso señala el resurgimiento de la movilización masiva de ciudadanos en Estados Unidos y otros países industrializados (Smith, 2002, 209). El tercer período inicia en el año 2001, concretamente a partir de los sucesos del 11 de septiembre, y se caracteriza por el aumento del control de la protesta social, la disminución de los espacios transnacionales de lucha y el repliegue de las instituciones multilaterales a espacios "inaccesibles".

Los movimientos sociales, por consiguiente, se encontrarían en una fase en la que no han logrado una unidad en las propuestas y alternativas, donde prevalecería la división entre movimientos del Norte $y$ del Sur, entre reformistas, transformacionales y de retorno a lo nacional (Ayres, 2005; Bello, 2004; Scholte, 2005), de lucha contra el militarismo hegemónico de un Estado (Tarrow, 2005; Wood, 2005) o de reconstrucciones de identidad y unidad de las luchas de reconocimiento y distribución (Fraser, 2003; Reitan, 2007). Este período se caracteriza asimismo por la prevalencia de una dicotomía en-

2 En este trabajo se entenderá por neoliberalismo la gestión global del poder político bajo principios de la economía de mercado (Foucault, 2004, 137). Lo anterior implica que estos mismos principios sean los reguladores del Estado, en tanto que formación jurídica y condensación de las relaciones sociales (Poulantzas, 1971). 
tre formas de acción colectiva, violentas o pacíficas, bien sea como opción estratégica o recurso táctico (Borras, 2008; della Porta, Andretta, Mosca, \& Reiter, 2006).

\section{5 ¿Qué están creando los movimientos sociales transnacionales?}

Más allá de la periodización que delimita sus finalidades y características, la cuestión central radica en establecer qué están haciendo dichos movimientos. La proposición es que éstos se hallan abocados a la construcción, desde los años 70 y paralelamente con todos los otros agentes no estales, de una sociedad civil global o internacional definida por interrelaciones complejas que, en alguna medida, estarían unificando un campo de acción social. En este campo, la sociedad civil global no se opondría a un Estado global (inexistente), ${ }^{3}$ sino que tendría un doble frente: los Estados nacionales y sus instituciones intergubernamentales y los organismos multilaterales.

Esta sociedad civil denotaría la existencia de intereses contradictorios, en particular, la consolidación de un cosmopolitismo según el cual todos compartiríamos ciertos problemas comunes y elementos esenciales en tanto seres humanos, es decir, se conservaría la idea de un sujeto universal con iguales potencialidades de transformación, independientemente de su contexto social. Este cosmopolitismo implicaría un fuerte arraigo social en lo local que uniría la prosecución de la solución de los problemas locales, en una lucha contra oponentes externos, pero impulsada por la búsqueda del bien común, lo cual conlleva un cambio de escala en la acción colectiva (Cohen \& Rai, 2000; Tarrow, 2005).

Otros autores, por el contrario, han expuesto la necesidad de asumir una renuncia total a los proyectos de vida y organización delimitados por lo local y lo nacional (Webster, 2004) y se inclinan en vez por plantear la existencia de un espacio social, más o menos unificado y nivelado, donde los movimientos sociales deberían contribuir a la solución de los problemas globales, a la transformación y monitoreo de las políticas de los Estados o de los organismos multilaterales y al proyecto de la globalización (Giugni, 2004; Kriesberg, 1997; O’Brien, Goetz, Scholte \& Williams, 2000; Podobnik, 2005; Smith, 1997).

Como ejemplos ilustrativos de lo expuesto podemos citar la Asociación de Mujeres Auto-empleadas de la India, que genera formas de empleo para las mujeres al margen del Estado, y la Internacional de Barrios/Tugurios (Slum/ShackDwellers International), cuyo objetivo es proveer soluciones a los problemas de habitación para pobladores pobres. Ambos movimientos tienen un propósito común, cual es el de reducir o menguar la vulnerabilidad de los sectores de base frente a las políticas globales y los cambios económicos (Batliwala, 2001, 394). En este mismo sentido cabe relevar los movimientos ambientalistas de los países del Norte que logran introducir reformas a las legislaciones internacionales a fin de salvaguardar el medio ambiente.

No obstante, la pregunta que urge resolver es desde dónde se ubican o definen los problemas que requieren una solución. Al respecto, surge una doble moción: de un lado, figuran los problemas planteados por esa sociedad civil global, específicamente por los movimientos sociales, cual es el caso concreto de la Vía Campesina y las problemáticas agrícolas locales que le dieron origen y propenden, ante todo, por una propuesta de desarrollo; y del otro, aquellos formulados por los organismos multilaterales y otras instituciones con el fin de salvar al "capitalismo de sí mismo", para lo cual se necesita una sociedad civil, vacía de las relaciones sociales y de las relaciones de fuerza implicadas en los problemas, que ayude, también, a legitimar la acción y existencia, tanto de dichas organizaciones multilaterales como del orden económico mundial (Drainville, 2004; Munck, 2007).

\footnotetext{
3 Aunque algunos autores como Hardt \& Negri (2000) plantean la existencia de una superestructura denominada "Imperio" que podría hacer las veces de gran Estado.
} 
En este sentido, los movimientos así definidos serían denominados globales o internacionales pues están presentes en un espacio socio-económico unificado cuyo contexto es la economía mundial. En palabras de Alejandro Colás, tales movimientos hacen parte de una sociedad civil internacional que se puede definir así:

"espacio socio-económico internacional creado internacionalmente y dentro de los Estados por la expansión de las relaciones de producción capitalistas, donde los movimientos sociales modernos persiguen objetivos políticos específicos" (Colás, 2002, 50).

Asimismo, los movimientos serían ambivalentes pues se debatirían entre aceptar los términos de la globalización o rechazarla y retirarse de ella al espacio nacional (Brecher, Costello, \& Smith, 2000, 16). Es por esta razón, precisamente, que se les conoce también con las denominaciones unívocas de movimientos anti-globalización o anti-sistema, contra-movimientos de la globalización o movimientos de protesta de la globalización; entre estos últimos se debe incluir, por igual, a los así llamados movimientos "reaccionarios", como los grupos de supremacía blanca norteamericanos y algunas agrupaciones nacionalistas que sienten amenazadas y debilitadas las estructuras sociales nacionales, la cultura y la soberanía del Estado por obra de los procesos de globalización (Castells, 1999; Munck, 2007).

A manera de recapitulación, puede decirse que los movimientos son globales cuando son supranacionales y sus causas han sido definidas o identificadas como globales (della Porta, et al., 2006, 18), o cuando actúan localmente y piensan globalmente (Munck, 2007). Por tanto, no basta con afirmar que un movimiento cruza las fronteras, moviliza sus constituyentes en más de dos Estados o se encuentra inmerso en una sociedad civil global, sino que se requiere investigar, tal y como lo proponen Della Porta y Tarrow (2005a, 232), las relaciones entre la lucha nacional y la contienda transnacional, es decir, indagar cuáles son las condiciones de posibilidad para que un movimiento surja como un agente social allende el nicho local.

\section{Globalización y movimientos sociales transnacionales}

El estudio de los movimientos sociales transnacionales ha adquirido mayor relevancia cuando se lo conecta con la denominada globalización o, en términos de algunos autores, con el proyecto neoliberal denominado globalización (Cfr. entre otros Gledhill, 2004; McMichael, 2004), proyecto que tiende a eliminar las condiciones de reproducción social de ciertos grupos a través de un movimiento de expropiación de las condiciones básicas de ser y existir de éstos, la reducción de los salarios, el deterioro de las condiciones laborales de los trabajadores, la supresión de servicios sociales en barrios, la expropiación de la tierra, semillas y conocimientos de los campesinos, entre otros. En otras palabras, nos encontramos en el período histórico descrito por Karl Polanyi, aquel que marca la transformación de la economía mundial.

La economía mundial y el sistema de relaciones internacionales se cimentaron, hasta el año 1914, en cuatro instituciones fundamentales, a saber: el patrón oro, el sistema de regulación de los conflictos entre las grandes potencias o sistema de balance del poder entre éstas, el mercado autorregulado y el Estado liberal (Polanyi, 2001, 3). La paz que duró desde 1815 hasta 1914 —paz de los 100 años, derrota de Napoleón, Congreso de Viena (restablecimiento de las monarquías y las fronteras), Tratado de Paris de 1889 que puso fin a la guerra hispanoestadounidense - contribuyó a establecer las bases de seguridad y soberanía que regirían subsecuentemente el sistema internacional.

La ruptura de este orden, por causa de la segunda conflagración mundial, pudo ser recompuesta en alguna medida y no sin controversia a través del sistema de Bretton Woods - patrón divisa (dólar estadounidense), Fondo Monetario Internacional, Acuerdo General sobre Tarifas y Aranceles (por su sigla en inglés GATT), Banco Mundial, Organización de las Naciones Unidas, Pax Americana-, el cual se quebró en 1971 como consecuencia de la declaración de Nixon respecto de la no convertibilidad del dólar en oro. Así se arriba al momento donde el Estado deja de ser el principio organizador 
de la economía y el capital se convierte en el nuevo principio ordenador y regulador de la economía y la sociedad (McMichael, 2008).

\section{El problema del desarrollo}

La fuerza dominante en el espacio internacional y global es el capital. Es éste, el que ha transformado el proyecto de desarrollo que había comenzado con el colonialismo - con cambios en el post-colonialismo - en un proyecto global. Esto significa que se ha dejado de concebir el desarrollo como un fenómeno centrado en la esfera nacional, es decir, bajo el modelo de crecimiento industrial, la expansión de los mercados y la regulación de los efectos socialmente indeseados. En el plano ideal, dicho modelo debería estar acompañado de una serie de instituciones de orden político que permitiese a los sujetos ser ciudadanos en una sociedad enmarcada dentro del llamado Estado nacional con plena soberanía. El modelo de desarrollo global se fundamenta, por el contrario, en el crecimiento económico organizado globalmente y su éxito se mide por la participación e integración en el mercado mundial; por tanto, las unidades territoriales del despliegue de este proceso pueden ser las ciudades, las bio-regiones, las redes de comercio, las cadenas de mercancías y las macro-regiones (McMichael, 2004, 154-155).

Cuando se plantea el desarrollo centrado en el crecimiento económico organizado globalmente, las atribuciones y soberanía del Estado nacional tienden, por consiguiente, a transformarse. Para que su acción contribuya al crecimiento económico, es preciso redefinir su rol y áreas de competencia, lo cual obliga, por igual, a modificar o alterar las fronteras, no necesariamente las geográficas pero sí las sociales, raciales y de género, entre otras. De este modo, se genera una ruptura en la congruencia para la acción política que planteaban los Estados, pues muchos de los procesos no son determinados enteramente en el plano internacional ni en el plano nacional, sino en la relación de ambos, en las vinculaciones de múltiples espacios, nacionales y globales. Según Alvater $(2002,49)$, esto lleva a la reducción de los espacios y los tiempos destina- dos a la cooperación social, es decir, menoscaba la expresión de la democracia formal. Sin embargo, lo dicho no significa que el Estado se encuentre en peligro de desaparecer o que, necesariamente, pierda eficacia, puesto que sigue proporcionando las condiciones materiales para la existencia social, lo cual permite a su vez la instauración de procesos de crecimiento globalmente orientados. En este sentido, es improcedente ubicar las relaciones de poder y fuerza totalmente por fuera del ámbito del Estado soberano (Mann, 2002, 177). Por consiguiente, cualquier estudio sobre los movimientos sociales debe ubicarse en un espacio que pueda relacionar y dar cuenta de los distintos niveles en que se presentan las fuerzas económicas y sociales, no como algo exterior o interno, sino donde sea posible aprehender su interrelación, e igualmente, sea dado reconocer las autonomías locales no necesariamente interrelacionadas con los procesos del capitalismo global.

\section{Lugar de los movimientos sociales}

El modelo de los movimientos sociales transnacionales fue formalizado por SidneyTarrow a partir de su modelo de los movimientos sociales nacionales (cfr. Tarrow, 1994). Así, se considera que el crecimiento de las oportunidades políticas fuera del Estado nacional, la incapacidad de éste de constreñir los movimientos sociales a las formas habituales, la declinación del poder estatal para controlar las fuerzas económicas globales —con el consiguiente aumento del acceso a nuevos recursos por parte de los movimientos sociales para aumentar su acción colectiva-y el surgimiento de algo parecido a una sociedad civil global, son los elementos cruciales que posibilitan la existencia de la acción colectiva transnacional (Tarrow, 1998, 233-234).

Si en un momento dado se llegó a pensar que la globalización y el desarrollo de los medios de comunicación y de transporte explicaban el surgimiento de los movimientos sociales transnacionales, esto se debió a un ilimitada fe en el poder de la tecnología y al desconocimiento de sus antecedentes históricos, pues estos medios no remplazan la organización ni las redes personales. Además, tal pensamiento 
olvida que muchos movimientos sociales previos surgieron y pudieron existir en la medida que no representaban un peligro real para el sistema estatal (Hanagan, 2002). La globalización en sí misma no explica ni remplaza a la organización de un movimiento, pero contribuye a generar mayores oportunidades porque, de una parte, aporta elementos para establecer relaciones entre diferentes grupos humanos que se sienten afectados por sus consecuencias y, por otra, ha hecho más visible una serie de instituciones a las que se ha responsabilizado de llevar a cabo las políticas neoliberales que tienen consecuencias negativas para los grupos sociales ajenos a las élites.

Sin embargo, se sigue considerando que el sitio por excelencia de disputa continúa siendo el Estado nacional, dado que éste no es, ni mucho menos, un ente pasivo ante los procesos de la globalización; por el contrario, es copartícipe en las formas que toma aquella en los distintos niveles de acuerdo con su posición en el sistema global de poder. Así, la cuestión que surge es cómo afectan los procesos de globalización la arena política nacional. El primer efecto es la disminución de la responsabilidad democrática del Estado, ya que éste no respondería al mandato de sus ciudadanos, sino, ante todo, a los designios de organismos multilaterales y corporaciones, entre otros, que formulan ciertas políticas por fuera del espacio social nacional. Por consiguiente, algunos agentes sociales, en particular, se encontrarían imposibilitados para alcanzar sus objetivos políticos si solamente realizaran su acción colectiva dentro de las fronteras nacionales, lo cual no soslaya el hecho de que son, en efecto, actores relativamente marginales porque se hallan excluidos del sistema de decisiones, tanto nacional como transnacional ${ }^{4}$ (Smith, 1997, 59; 2005, 32).

Lo dicho significa que estos movimientos sociales no pueden desvincularse por completo del espacio nacional, porque su poder reside, precisamente, en la capacidad de movilizar sus constituyentes, los cuales existen y viven en unas condiciones sociales determinadas que se distinguen por su falta de uniformidad e igualdad en los distintos niveles (local, nacional y transnacional).

Entonces, más que la globalización en general, es la globalización neoliberal la que proveería los "contenidos" de controversia en la medida que ha sido esta última la artífice del cambio de paradigma del desarrollo, es decir, ha promovido el modelo ya descrito que se centra en la esfera internacional y compele al desmonte de las estructuras del Estado de Bienestar. Medidas como las anteriores son resentidas por los grupos "subordinados" como una violencia estructural ya que destruyen las condiciones sociales de reproducción de ciertos grupos sociales, por ejemplo los campesinos, y los somete a otras que no responden a sus proyectos de vida, cultura y economía, lo que crea "un registro de sufrimiento" para tomar las palabras de Isabel Sommier (2003, 231).

El proyecto de la globalización no se ha desenvuelto empero sin oposición ya que, como argumenta Ronaldo Munck (2007), el planteamiento de la globalización neoliberal, según el cual el mercado autorregulado es el garante del bienestar social, ha creado una crisis generalizada. Es por consiguiente labor de la gobernanza global, condensada en una serie de instituciones tales como la Organización Mundial del Comercio (OMC), el Banco Mundial, etcétera, el morigerar los excesos del capital. Sin embargo, para algunos movimientos sociales, aquellas no son más que las representantes de las grandes corporaciones, los agentes que mayores beneficios han obtenido en virtud de la globalización de las relaciones sociales de producción capitalistas. Así, es el multilateralismo el que presentaría una serie de espacios para la acción colectiva de los movimientos sociales transnacionales, espacios que se habrían abierto tanto por la iniciativa propia de estas instituciones, en sus deseos de legitimar los procesos que impulsan, así como por la lucha de los movimientos sociales y otros agentes. En esta dialéctica se presentan las intervenciones de los movimientos sociales como la Vía Campesina, que han exigido la

4 Esto debe ser matizado ya que los procesos de la gobernanza global han llevado a que estén presentes en muchos lugares de decisión, aunque de manera controlada o restringida. 
participación en estos espacios no sólo con voz sino con poder de decisión.

En la medida en que dichos organismos han recogido las exigencias de los movimientos, se ha logrado mantener un diálogo. Aun así, los problemas abordados cambian de una institución a otra y el grado de apertura de cada una ha sido disímil, hecho que se ha justificado aduciendo la alta complejidad y tecnicidad de los temas a tratar, por ejemplo, la inflación, los flujos monetarios internacionales, las tasas de cambio, entre otros (O'Brien, et al., 2000). Sin embargo, según se afirmó, el terreno privilegiado de los movimientos sociales transnacionales continúa siendo el Estado y las instituciones de la gobernanza global. Éstas, en particular, son utilizadas, a veces, para inducir o lograr modificaciones en las políticas públicas nacionales, como ha sido el caso de la Organización Internacional del Trabajo (OIT). Por mediación suya, los movimientos de trabajadores de los países desarrollados y no desarrollados han querido imponer las normas laborales aplicadas en los países del centro a los países de la periferia. En el sector agropecuario, por su parte, se ha producido una confrontación intensa con la finalidad de forzar a los Estados a prohibir las patentes de material vivo y limitar la injerencia de la Organización Mundial del Comercio en el tema por medio de regulaciones comerciales y derechos intelectuales; asimismo, se ha buscado el retiro de aquél de su ámbito regulatorio.

Los movimientos sociales transnacionales se encuentran, por consiguiente, en capacidad de unir tanto los distintos recursos institucionales y materiales (medios financieros, bienes y servicios),como la acción misma de los propios constituyentes, a fin de modificar y transformar los espacios y políticas objeto de sus luchas. Al contrario de lo afirmado por Sommier (2003, 232), para quien éstos son movidos por un sentimiento de impotencia política o difícilmente pueden ofrecer algo en una negociación a cambio de las concesiones que se les dé (Bonnassies, 2005, 37), no sólo han demostrado que tienen la capacidad de reunir recursos y generar alternativas viables $-\mathrm{y}$, de ser necesario, incluso, proveer servicios sin los cuales los Estados y los organismos multilaterales se verían abocados a enfrentar nuevos problemas para la realización de sus proyectos-, sino que, por sobre todo, detentan el suficiente poder para detener el proceso de globalización y deslegitimar o legitimar, según el caso, a ciertas instituciones (Bello, 2004; Brecher, et al., 2000, 21).

Tal proceso no es sin embargo mecánico, porque depende de la coyuntura, de la organización y coordinación del movimiento, de las alianzas, de los adversarios (Estados, organismos multilaterales e intergubernamentales, corporaciones multinacionales), de la correlación de fuerzas y de las luchas que se instauren en espacios sociales que exigen tácticas y estrategias específicas (acción directa, violenta, pacífica, de legitimación o de deslegitimación). En síntesis, la globalización es el fulcro para las luchas, pero al mismo tiempo constituye la trama que conjuga las fuerzas impulsoras de la disposición del capital a escala mundial, lo cual no significa que se trate de un espacio dado sino de un ámbito sujeto a incesantes reconfiguraciones.

\section{La gobernanza global}

Al formularse los procesos del capital a nivel mundial, junto con su proyecto de desarrollo global, se hace necesario, como afirma Drainville (2004, 106), insuflarle vida a este proceso a través de la construcción de un espacio cívico perfectamente apolítico, en el cual estaría presente una serie de agentes sociales acogidos bajo el título de "sociedad civil global", con el objeto de que contribuya al ordenamiento del espacio de la economía planetaria y con la cual sea posible negociar las reglas para el crecimiento económico. Dicha sociedad civil internacional sería relevada por un proceso denominado gobernanza global o:

"sistema de reglas e instituciones establecido por la comunidad internacional y los actores privados para administrar los asuntos políticos, económicos y sociales" (World Commission on the Social Dimension of Globalization, 2004, 75). 
Para su implementación, se requiere del concurso no sólo de los Estados y de las organizaciones internacionales sino de todos aquellos que puedan y deban contribuir tanto a la solución de los problemas como a la administración que éste demande.

Cynthia Hewitt de Alcántara (1998, 106107) afirma que el concepto de gobernanza, desarrollado inicialmente por la instituciones financieras internacionales en la década de los 70 , les ha permitido a estas instituciones intervenir en una serie de dominios, políticos y sociales, que antes les eran prohibidos, sin antagonizar directamente con los Estados. Este concepto es compartido por toda una serie de instituciones y no solamente las financieras comprometidas en la regulación de la economía global y el espacio transnacional con cierta autonomía respecto de los Estados. En consecuencia, permite intervenir en diversos asuntos que antes eran considerados como competencia exclusiva del Estado, al igual que en una serie de cuestiones que solía escapar su control pero en la cual tenía mayor o menor incidencia.

La gobernanza global implica pues el sometimiento de las políticas nacionales a un sistema de reglas, de sistemas legales que, en cierta forma, sobrepasan la soberanía nacional y tiene por objeto reforzar la economía global en sus circuitos de dinero, mercancías y capital. La mayor parte de normas provienen de los organismos financieros multilaterales, grupos de países desarrollados, corporaciones transnacionales y son asumidas con variaciones por los distintos Estados nacionales (McMichael, 2004, 139; Sassen, 1996, 29). A este sistema de gobernanza se ha invitado a participar a distintos movimientos sociales, algunos de los cuales han permanecido durante algún tiempo en los espacios propios de su esfera. Sin embargo, algunos han terminado retirándose, lo cual, contrario a lo que podría suponerse, no implica necesariamente el retraimiento a un "espacio interior", sino que expresa la búsqueda de un replanteamiento de las condiciones generales de su existencia. De este modo estarían ejerciendo lo que podría calificarse de un tipo de resistencia, entre múltiples formas y espacios de resistencia, consistente en el rechazo de las normas e instituciones hegemónicas (van der Linden, 2003, 25) en las cuales se hallan afincados los procesos de globalización neoliberal.

Es preciso, por tanto, prestar especial atención a la articulación de las formas de resistencia que puedan darse en los espacios locales, regionales y transnacionales, porque cualquier forma de rechazo a las instituciones globales y sus normas debe tomarse como un signo de la existencia de otros procesos de resistencia en otros espacios, especialmente si tomamos en cuenta la limitada movilidad territorial de los constituyentes de estos movimientos. En otras palabras, la puesta en cuestión que realiza un movimiento social del modelo de desarrollo global es viable siempre y cuando se sustente en procesos locales. No es dado entonces plantear que los procesos de cambios de escala de la acción colectiva se realizan ante el simple cierre de las oportunidades nacionales para la acción. Es precisamente en este punto donde el concepto de espacio social transnacional sirve de instrumento de investigación fundamental en la medida que permite ubicar las luchas y los objetivos de cada movimiento en una trama conceptual que arroja luces sobre su articulación y reduce la arbitrariedad en la interpretación de la acción colectiva.

Así, podemos plantear, primero, que la globalización - toda definición de globalización que tomemos será necesariamente incompleta y objeto de controversia- se refiere al proceso histórico de acumulación y restructuración del capital a escala planetaria $y$, segundo, que dicho proceso entraña una tensión entre el desacoplamiento de la economía de las normas sociales y la formulación de sistemas de reglas en aras de garantizar el crecimiento y la acumulación. La globalización expresa, por consiguiente, las relaciones sociales que enuncian, en palabras de Boaventura de Sousa Santos (2003, 226), la aceleración de los intercambios transnacionales. En este sentido, los movimientos sociales transnacionales están contribuyendo a darles forma porque representan las condiciones materiales $y$ discursivas en contra de las cuales se plantea la acción de los organismos multinacionales $e$ insti- 
tuciones que impulsan el desarrollo global (McMichael, 2004, 238). La globalización representa las condiciones generales aptas para el surgimiento de los espacios sociales transnacionales; sin embargo, una vez constituidos, sus relaciones con esas condiciones generales se modifican y éstas, a su vez, mudan a su efecto.

\section{La articulación de los movimientos sociales transnacionales}

La literatura científica relativa a los movimientos sociales transnacionales ha tendido a dar por supuesto que estos son de por sí sujetos colectivos políticos. ${ }^{5}$ De este modo se trataba de asir y describir, entre otros temas, los procesos utilizados para trasladar de un espacio social generalmente nacional a uno transnacional, las oportunidades de la acción de los movimientos sociales y el activismo transnacional (della Porta \& Tarrow, 2005b; Giugni, 2004; McCarthy, 1997; Reitan, 2007; Smith, 2005; Tarrow, 2005).

El mismo tipo de razonamiento está presente en la caracterización de las sociedades latinoamericanas ${ }^{6}$ $y$ de los movimientos sociales surgidos en ellas, es decir, se da por hecho el paso de una sociedad rural a una urbana e industrial (Foweraker, 1995, 5) y tal proceso explicaría tanto la aparición de nuevos sujetos políticos como la destitución o, al menos, la disminución del peso específico de otros sujetos, verbigracia, los campesinos. Sin embargo, este supuesto paso no era sometido a un trabajo de conceptualización porque la urbanización y la industrialización, aparentemente, marcaban de hecho el carácter de las sociedades afectadas. De esta guisa se dejaba de lado la pregunta sobre los determinantes sociales, políticos y económicos que definen una sociedad dada, como los movimientos poblacionales, el peso específico de las poblaciones rurales -independientemente de su número relativo-y del sector rural (no solo agrario, sino minero y biodiverso, entre otros) en la economía, y el origen y dinámicas de los conflictos sociales fundamentales a cada sociedad. Del mismo modo se daba por hecho que los flujos de información y la automatización determinaban de por sí al capitalismo, tanto como las relaciones sociales generadas por éste, y el grado de importancia del Estado (Castells, 1999).

Plantear la cuestión teórica de la importancia de un movimiento social para la constitución de los sujetos políticos transnacionales implica igualmente discernir qué es lo que determina a un movimiento social - asible a través de una organización del movimiento social- como local y/o nacional; cuáles son las características de su "agenciamiento" o acción orientada; y sus formas de articulación. Así, la existencia de los movimientos en un espacio social distinto al local y al nacional no conlleva per se que se trate necesariamente de movimientos transnacionales. Según sostienen Khagram et al. (2002, 5), es necesario aún tender puentes entre la teoría de los movimientos sociales transnacionales y las teorías sociológicas y políticas (Khagram, et al., Por consiguiente, si aceptamos como punto de partida que sólo en el espacio local-nacional "son sujetos históricos en el sentido completo del término" y reconocemos que:

"En la economía mundial hay sujetos abstractos, portadores de todo tipo de maneras y estrategias, pero sin construirse políticamente" (Drainville, 2004, 24).

Debemos concluir que en el ámbito global:

"Los sujetos transnacionales no tienen ningún lugar propio, con excepción de los conjuntos idealizados u objetivados" o "la familia del mundo de los movimientos antisistémicos"(Drainville, 2004, 25).

Lo expuesto nos remite de nuevo al espacio social local-nacional y es en esta esfera donde cabe pre-

\footnotetext{
De acuerdo con algunas de las teorías, al menos sus componentes singulares e individuales.

6 Si bien lo enunciado no se profundizará en el presente estudio, el análisis de los movimientos sociales transnacionales sólo adquiere sentido si se relaciona con condiciones sociales concretas.
} 
guntarse si estos movimientos sociales son sujetos políticos por el solo hecho de realizar una serie de demandas y acciones colectivas o si se requiere de un elemento de transcendencia que logre contribuir a su definición veraz de sujetos políticos. De este modo es posible evitar lo que, por ejemplo, Alison Brysk denomina movimientos sociales campesinos con carácter étnico (ethnicizedpeasantmovements), es decir, aquellos levemente internacionalizados y cuyas demandas se habían circunscrito hasta hace poco tiempo al ámbito económico y han sido luego extendidas a los derechos tradicionales sobre la tierra y a las preocupaciones culturales (Brysk, 2000, 71-72).

Ahora bien, lo que realmente puede posibilitar la precipitación de un movimiento social en sujeto político es, en palabras de Foweraker, la unión de las preocupaciones sociales y privadas con las políticas institucionales y estatales vigentes. Así, la posibilidad de que un movimiento social logre esta expresión se conjuga en un proyecto político (Foweraker, $1995,62) .{ }^{7}$ Es pues en relación con el Estado que se constituye la esfera política y es en los distintos niveles que éste brinda donde es posible lograr que los sujetos sociales adquieran un carácter político.

De acuerdo con Hobsbawn, la existencia de movimientos, de luchas en el ámbito local, hace que nos encontremos en un ámbito pre-político, en la medida que no es sólo una relación con las políticas y el Estado lo que establece la posibilidad de adquirir el carácter político que determinaría a estos sujetos sino es la relación con un ámbito nacional político-estatal lo que marca la política moderna (Hobsbawm, 2001) y, por consiguiente, la constitución de los sujetos políticos. Así que si nos preguntamos cuál es la importancia de un movimiento social para la constitución de los sujetos políticos transnacionales, la respuesta necesariamente pasa por indagar su relación con la constitución de los sujetos políti- cos locales y nacionales. En otras palabras, se trata de dilucidar si el movimiento real que constituye va de lo local a lo nacional y de lo nacional a lo transnacional-internacional, o si da un salto directo de lo local hacia el ámbito transnacional.

Lo anterior nos remite a la pregunta relativa a la diferencia entre un movimiento social local y uno nacional. $\mathrm{Al}$ interrogante podemos responder que la diferencia compete a la relación que se haya establecido con las instancias de poder político, especialmente el Estado. Ahora bien, cuando se refiere al Estado, no se trata en este caso de la simple distribución de los recursos estatales, o la participación en instancias consultivas, sino del establecimiento de relaciones de poder que permitan modificar el sentido y desenvolvimiento de las políticas y acciones de aquél. Así, un movimiento local estará restringido al ámbito pre-político, del que habla Hobsbawn, si sus acciones son reivindicativas y no propositivas y/o proyectivas - en el sentido de Foweraker-e igualmente cuando la actividad orientada busque cambiar tan solo las desigualdades y matizar los contrastes territoriales ${ }^{8}$.

Por oposición, el movimiento nacional se definiría en términos negativos con respecto a lo local. Esto significa que su "agenciamiento" perseguiría más el control del poder político y del aparato del Estado por medio de la acción directa y en una dinámica de integración y confrontación con los partidos políticos que actúan a través de la representación (Foweraker, 2001, 8). Por otra parte, un movimiento local requeriría convertirse en nacional si tiene la pretensión de superar tanto las condiciones generales como la debilidad resultante de la alta movilidad de sus constituyentes - fuerza laboral, migraciones forzadas, entre otros-(Brittain, 2005), y si se consideran a la par los lazos, las alianzas y los tipos de organización de los agentes contrincantes que actúan por lo general en un contexto más amplio

7 La referencia a los movimientos campesinos de carácter étnico, enunciada por A. Brysk, o aquellos que reivindican derechos ancestrales y culturales, connota la presencia de un tercero a quién se le puede exigir servir de garante de los mismos, corrientemente, el Estado. Por tanto, es en relación con este último que un movimiento campesino puede asumirse como sujeto político.

8 No obstante, la relación con el Estado están mediadas por la "distancia" de los movimientos sociales con él, ya sea en términos geográficos, institucionales, culturales y/o de clase; de esta forma, no podría hablarse de espacios pre-políticos. Al definirse la distancia con el Estado, ésta tendría de por sí un sentido político (Davis, 1999). 
y con múltiples conexiones con factores de poder (van der Linden, 2003, 12). Así, un movimiento que se provea de una estrategia nacional ( $\mathrm{y} / \mathrm{o}$ internacional) debe dotarse a la par de una serie de cuadros móviles y permanentes en torno a los cuales se formen las fuerzas y movimientos locales (Hobsbawm, 1999, 172), es decir, dicha serie contribuiría a quebrar las condiciones que determinan, por lo general, a los constituyentes de los movimientos y las organizaciones.

De manera simplificada, parte de la literatura científica aquí tratada ${ }^{9}$ supone que el paso de lo local a lo nacional es la forma de constitución del sujeto político de los movimientos sociales. Este mismo argumento podría extenderse para cubrir el paso de los movimientos sociales nacionales a lo transnacional o internacional, pues las condiciones generadas por la globalización harían de lo nacional un equivalente de lo local y colocarían a lo internacional como el término correspondiente a lo nacional. En otras palabras, un movimiento social nacional tiene como constituyentes movimientos y organizaciones locales que se transforman en tanto adquieren un horizonte diferente al entrar en el terreno definido por el Estado, y si cuestionan este espacio y las relaciones sociales allí establecidas.

La existencia de un movimiento nacional no implica, necesariamente, que éste sea un sujeto político en sentido pleno de la palabra, puesto que puede ser el objeto de las acciones de otros sujetos. Lo expuesto puede ilustrarse con la historia de la Asociación Nacional de Usuarios Campesinos (ANUC), organización que fue creada y financiada en parte por el Estado colombiano. Así, las organizaciones campesinas y el movimiento campesino eran objeto de las políticas, programas y proyectos estatales y sus componentes individuales y colectivos eran pensados, definidos y tratados como usuarios de los servicios ofrecidos por aquél para el sector rural. En síntesis, eran objeto del Estado como única posibilidad de evi- tar que se convirtiesen en sujetos políticos con capacidad para cuestionar el orden imperante (Múnera Ruiz, 1998; Zamosc, 2001). Más adelante, cuando la ANUC logró reflexionar autónomamente respecto de su quehacer y de sus relaciones con el Estado, y asumió en consecuencia posiciones y formas de lucha más beligerantes, se convirtió en "enemigo" o sujeto oponente que amenazaba la unidad y la comunidad nacional (Schmitt, 1990), comunidad definida desde los términos del poder y encarnada en los dos partidos tradicionales: el Liberal y el Conservador.

La existencia de un movimiento social transnacional no es de por sí indicio de que equivalga aun sujeto político transnacional o contribuya a la constitución de sujetos de este orden. Organizaciones como el Foro Social Mundial, la Vía Campesina, compuestas de movimientos sociales locales, nacionales y de organizaciones que podrían denominarse internacionales no pasan por la conceptualización que rastreamos brevemente al abordar el proceso de constitución de los movimientos sociales en sujeto políticos nacionales. Es, al parecer, su "entrada" y articulación en otro espacio lo que señala en parte su formación como sujetos políticos.

En síntesis, de una parte se ha planteado que la posibilidad de constitución de los movimientos sociales en sujetos políticos pasa por las relaciones con el Estado y que tal trato ha de transcender al ámbito nacional para adquirir un pleno sentido, pues es allí donde se define lo político en términos modernos (Koalka, 1966, 211), y de otra, que la sola existencia de movimientos sociales que actúan a nivel nacional y sostienen relaciones con el Estado no basta para calificarlos de sujetos políticos, así éstos sean muchas veces objetos de políticas por parte de aquél. De seguir la reflexión propuesta por Marx (1987), el Estado sería la superficie de constitución de los sujetos políticos puesto que genera las condiciones generales para el desenvolvimiento de los sujetos. Esto sería cierto si el Estado se presentase

9 La revisión de la literatura científica realizada en este texto ha hecho un mayor énfasis en las fuentes teóricas que han permitido conceptualizar el surgimiento y trayectoria de los movimientos sociales transnacionales. No obstante, es necesario subrayar la existencia de una gran cantidad de producción en español que da cuenta del papel de estos movimientos en Iberoamérica y el Caribe (Véase entre otros: Arias Maldonado, 2008; Evans, 2007; Korzeniewicz \& Smith, 2004). 
como un "igual" ideal frente a los movimientos y los distintos agentes sociales, es decir, sin intentar transformarlos en objetos de su poder. ${ }^{10}$ Es tan solo cuando los movimientos sociales aceptan cuestionar, e incluso transformarlas condiciones de poder fundamentales en el curso de su propia dinámica, según lo insinúa Foweraker (1995),que logran adquirir una relación de "igualdad" —al menos imaginaria- con el Estado.

No obstante, movimientos como la Vía Campesina Internacional muestran que, tal cual sucede con la formación de los movimientos sociales nacionales, la constitución de un sujeto político transnacional ya no necesita pasar directamente por el Estado sino por relaciones en otro terreno distinto al nacional y con respecto a otras instancias y otros sujetos sociales, entre los cuales figurarían tanto el propio Estado nacional como los organismos de la gobernanza global.

Si pudiésemos resumir en una expresión cuál es la dinámica presente, podríamos decir que nos encontramos en presencia de un movimiento de ida $y$ vuelta en el cual lo exterior se encuentra en el interior (Lacan, 1973, 165) y esto es lo que permitirá la articulación ${ }^{11}$, mas ya no concebida como la unión entre elementos separados o desagregados sino cual la dinámica que cambia los lugares y posiciones de los elementos creando nuevas condiciones sociales y políticas. ${ }^{12}$ Por tanto, los movimientos del orden local y nacional no se hallarían necesariamente en un espacio confinado dado que las fronteras han sido siempre relativamente porosas. ${ }^{13}$
La diferencia entre la acción social local y la acción social transnacional de los movimientos sociales sólo puede aprehenderse en la medida que el movimiento social transnacional opere en múltiples niveles; cada nivel implica un cambio en las condiciones, en los discursos, en el tipo de acciones del movimiento. De acuerdo con Brysk, lo anterior sigue, por lo general, la ruta causal que incluye el ejercicio de la autoridad, la circulación de personal, la transferencia de recursos, la influencia ideológica, la presencia y proximidad físicas y el respaldo de actores intermediarios (Brysk, 2000, 22-23). En consecuencia, puede afirmarse que se trata de una relación con la economía mundial, como espacio social contextual mayor en el cual se estarían definiendo las condiciones de la acumulación global del capital y donde residiría la especificidad de los movimientos sociales transnacionales. Estas relaciones son las que están en juego y pretenden regularse a través de una sociedad civil neutra o, en su remplazo, por la gobernanza global (Drainville, 2005).

No obstante, la limitación de la posibilidad de acceso a este espacio, el empleo de mayores medidas de control de la protesta social y el consecuente retiro de las instancias de la gobernanza global a fin de evitar encontrarse al alcance de los movimientos sociales y la sociedad civil, indica que la entrada a esta esfera se ha restringido a unos cuantos (della Porta, et al., 2006, 138), es decir, la economía mundial se ha convertido en: "Un espacio de exclusión" (Munck, 2004, 61).

Que puede poner en cuestión la regulación misma del capital. ¿Pero cuáles relaciones están estable-

10 La fuente es un texto de Carlos Marx donde afirma: "En cierto modo, con el hombre sucede lo mismo que con la mercancía. Como no viene al mundo con un espejo en la mano, ni tampoco afirmando, como el filósofo fitchiano, 'yo soy yo', el hombre se ve reflejado primero sólo en otro hombre. Tan sólo a través de la relación con el hombre Pablo como igual suyo, el hombre Pedro se relaciona consigo mismo como hombre. Pero con ello también el hombre Pablo, de pies a cabeza, en su corporeidad paulina, cuenta para Pedro como la forma en que se manifiesta el genus [género] hombre" (Marx, 1987, 65).

11 En términos operativos puede decirse, de acuerdo con Múnera, que "los componentes de un movimiento pueden integrarse entre sí, separarse temporal y parcialmente, o reintegrarse en una dinámica que coloca las acciones colectivasen un devenir y una transformación constante, debido a las relaciones internas entre los diferentes actores. Por consiguiente, dicha articulación debe ser comprendida como un proceso social y no como un simple hecho institucional" (Múnera Ruiz, 1998, 61).

12 Aquí nos referimos a la actividad con un fin de los movimientos sociales para nuestro caso y no a la combinatoria de elementos estructurales.

13 En este punto es necesaria una referencia al concepto de espacio social transnacional, en la medida que el espacio de la globalización se cerraría. 
ciendo los movimientos sociales que buscan constituir alternativas, o al menos redefinir, en parte, la economía mundial, y en dónde? Movimientos como la Vía Campesina Internacional están al presente poniendo en contacto espacios y lugares que antes se encontraban separados y tal quehacer cuestiona las relaciones sociales y el dispositivo que determina distintos sectores económicos y sociales. De este modo, se realiza una articulación que actúa como "cortocircuito" en tanto se superan mas no se borran las distinciones entre los diferentes niveles (Žižek, 2003, 238).

Lo anterior lo realiza, por ejemplo, la Coordinadora Latinoamericana de Organizaciones del Campo (CLOC, puesto que:

\section{"[...] Busca articular las organizaciones de masas campesinas. Siempre haciendo un mínimo de acuerdos que de hecho pu- diesen representar actividades concretas en la articulación en la solidaridad, en el intercambio, etc. La articulación [...] siem- pre tuvo un carácter ideológico, [...]. el carácter ideológico estuvo y está presen- te, pues prácticamente todas las organi- zaciones están ligadas a los movimientos de transformaciones sociales $y$, es claro, con sus formas de luchas, ligadas a la rea- lidad de cada país. [Entonces se requiere] un mínimo de articulación nacional de las organizaciones para evitar que en el país las organizaciones no se articulen y en el plano internacional, quieren (sic) hacer la revolución"(Coordinadora Latinoamerica- na de Organizaciones del Campo - CLOC, 2005, 58).}

\section{Conclusión}

Los movimientos sociales transnacionales surgen cuando comienzan a construir un espacio social propio de lucha social que no es el simplemente dado por la economía mundial en su etapa de la globalización neoliberal. En este sentido, ellos han formado y creado parte de las condiciones materiales que en algunos momentos han permitido el de- sarrollo y algunas transformaciones del proyecto de la globalización. Cuando los movimientos sociales transnacionales logran convertirse en agentes políticos (no meramente reproductivos), las relaciones con sus oponentes se transforman en virtud de ello. Así, verbigracia, los organismos de la gobernanza global se han visto obligados a realizar llamados para la construcción de una sociedad civil global que contribuya a la solución de los problemas globales que nos "atañen a todos como seres humanos", con la esperanza de eludir, al mismo tiempo, cualquier cuestionamiento sobre las condiciones y la formulación misma de estos problemas. Pero ya que la así denominada social civil global no ha logrado cristalizarse, sino su remedo a través de una serie de asociaciones o instituciones, se ha perfeccionado en vez un sistema de regulación de la economía mundial capitalista bajo el nombre de gobernanza global, el cual ha sido igualmente puesto en cuestión con consecuencias significativas: de un lado, ha forzado el retiro de sus instituciones bandera (FMI, Banco Mundial, Foro Económico Mundial, entre otras) a ámbitos inaccesibles; y del otro, ha promovido un mayor y más férreo control social y policial de todo movimiento social nacional o transnacional que se atreva a criticar o controvertir los términos de la globalización.

El anterior proceso señala igualmente una transformación de los movimientos sociales que trascienden el ámbito local y nacional. Desde el siglo XIX, los movimientos sociales internacionales o globales se plantearon en nombre de sujetos sociales generales que existían independientemente de las condiciones locales; al presente, se reconoce la existencia de unas condiciones generales y locales que establecen diferencias entre los distintos agentes sociales por el solo hecho de compartir unas dichas condiciones o algunas características particulares como son la clase, el género y la condición social, entre otras. Tal diferenciación permite introducir el problema de articulación de dichos movimientos y agentes o sujetos sociales. Ahora bien, no es posible afirmar la existencia de movimientos sociales globales, o que aspiran a serlo, sino de movimientos que coordinan demandas y luchas que ponen en cuestión tanto lo local como lo global y evidencian la contradicción y desfase entre las exigencias de la 
reproducción social y los imperativos de la acumulación capitalista.

\section{Referencias}

Altvater, E. (2002). El lugar y el tiempo de lo político bajo las condiciones de la globalización económica. [Ort und Zeit des politischen unter Bedingungen ökonomischer Globalisierung]. En: Zona Abierta(92/93): 7-60.

Arias, M. (2008). La globalización de los movimientos sociales y el orden liberal. Acción política, resistencia cívica, democracia. En: Revista Española de Investigaciones Sociológicas (Reis)(124): 11-44.

Ayres, J. (2005). From "Anti-Globalization" to the Global Justice Movement: Framing Collective Action against Neoliberalism. En B. Podobnik \& T. Reifer (Eds.), Transforming Globalization: Challenges and Opportunities in the Post 9/11 Era (927). Leiden: Brill.

Batliwala, S. (2001). Grassroots Movements as Transnational Actors: Implications for Global Civil Society. Voluntas: International Journal of Voluntary and Nonprofit Organizations, 13(4): 393-409.

Bello, W. (2004). Deglobalization: Ideas for a New World Economy (2nd ed.). London; New York: Zed Books.

Bohórquez, J. (2009). Transnacionalismo e historia transnacional del trabajo: Hacia una síntesis teórica. Papel Político, 14(1): 273-301.

Bonnassies, V. (2005). La transnationalisation des mouvements sociaux dans les Amériques et son impact sur la redéfinition du politique : vers une typologie (Vol. 05-02). Montréal: Centre Études internationales et Mondialisation. Borras, S. (2004). La Vía Campesina: Un movimiento en movimiento (B. Martínez Ruiz, trad.). Amsterdam: Transnational Institute.

Borras, S.(2008). La Vía Campesina and its Global Campaign for Agrarian Reform. Journal of Agrarian Change, 8(2-3): 258289

Brecher, J, Costello, T. \& Smith, B. (2000). Globalization from Below: The Power of Solidarity. Cambridge, Mass.: South End Press.

Brittain, J. (2005). A Theory of Accelerating Rural Violence: Lauchlin Currie's Role in Underdeveloping Colombia. Journal of Peasant Studies, 32(2): 335-360.

Brysk, A. (2000). From Tribal Village to Global Village: Indian Rights and International Relations in Latin America. Stanford Stanford University Press.

Castells, M. (1999). The Information Age: Economy, Society and Culture. Malden - Oxford: Blakwell Publishers.

Cohen, R., \& Rai, S. (2000). Global Social Movements: Toward a Cosmopolitan Politics. En: R. Cohen \& S. M. Rai (Eds.), Global Social Movements (1-17). London; New Brunswick, $\mathrm{NJ}$ : The Athlone Press.

Colás, A. (2002). International Civil Society: Social Movements in World Politics. Cambridge: Polity.

Coordinadora Latinoamericana de Organizaciones del Campo - CLOC. (2005). Directrices operativas y de funcionamiento de la CLOC. En: Coordinadora Latinoamericana de Organi- zaciones del Campo - CLOC (Ed.). Memoria IV Congreso Continental de la CLOC.

Davis, D. (1999). The Power of Distance: Re-Theorizing Social Movements in Latin America. Theory and Society, 28(4): 585638.

della Porta, D., Andretta, M., Mosca, L., \& Reiter, H. (2006). Globalization from Below: Transnational Activists and Protest Networks. Minneapolis; London: University of Minnesota Press.

della Porta, D., \& Tarrow, S. (2005a). Conclusion: "Globalization," Complex Internationalism, and Transnational Contention. En D. della Porta . \& S. Tarrow (Eds.). Transnational Protest and Global Activism ( 227-245). Lanham, MD: Rowman \& Littlefield Publishers.

della Porta, D., \& Tarrow, S. (2005b). Transnational Process and Social Activism: An Introduction. En D. della Porta \& S. Tarrow (Eds.). Transnational Protest and Global Activism (117). Lanham, MD: Rowman \& Littlefield Publishers.

Drainville, A. (2004). Contesting Globalization: Space and Place in the World Economy. London: Routledge.

Drainville, A. (2005). Québec city 2001 and the making of transnational subject. En: L. Amoore (Ed.), The Global Resistance Reader (169-189). London; New York: Routledge.

Evans, (2007). Instituciones y desarrollo en la era de la globalización neoliberal (C. F. Morales de Setién Ravin, Trans.) Bogotá: ILSA.

Faist, T. (2000). The Volume and Dynamics of International Migration and Transnational Social Spaces. Oxford: Claredon Press; Oxford University Press.

Foucault, M. (2004). Naissance de la biopolitique. Paris: École des hautes études en science sociales/Gallimard/Seuil.

Foweraker, J. (1995). Theorizing Social Movements. London: Pluto Press.

Foweraker, J. (2001). Grassroots Movements, Political Activism and Social Development in Latin America: A Comparison of Chile and Brazil. Geneva: USRID.

Fraser, N. (2003). Rethinking Recognition: Overcoming Displacement and Cultural Reification in Cultural Politics. En B. Hobson (Ed.), Recognition Struggles and Social Movements: Contested Identities, Agency and Power (21-32). Cambridge, UK: Cambridge University Press.

Giugni, M. (2004). Social Protest and Policy Change: Ecology, Antinuclear, and Peace Movements in Comparative Perspective. Lanham (Madison): Rowman \& Littlefield.

Gledhill, J. (2004). Neoliberalism. En: D. Nugent \& J. Vincent (Eds.), A Companion to the Anthropology of Politics (332348). Malden: Blackwell.

Hanagan, M. (2002). Irish Transnational Social Movements, Migrants, and the State System. En: J. Smith \& H. Johnston (Eds.), Globalization and Resistance: Transnational Dimensions of Social Movements (53-73). Lanham, Maryland: Rowman \& Littlefield.

Hardt, M., \& Negri, A. (2000). Empire. Cambridge: Harvard Universtiy Press.

Hewitt de Alcántara, C. (1998). Uses and Abuses of the Concept of Governance. International Social Science Journal, 155: 105-113.

Hobsbawm, E. (1999). Vietnam y la dinámica de la guerra de guerrillas (R. Pochtar, Trans.) Gente poco corriente. Resistencia, rebelión y jazz (169-181). Barcelona: Crítica.

Hobsbawm, E. (2001). Rebeldes primitivos. Barcelona: Crítica. 
Khagram, S., Riker, J., \& Sikkink, K. (2002). From Santiago to Seattle: Transnational Advocacy Groups: Restructuring World Politics. En: S. Khagram, J. V. Riker \& K. Sikkink (Eds.), Restructuring World Politics: Transnational Social Movements, Networks, and Norms (Vol. 1: 3-23). Minneapolis: University of Minnesota Press.

Koalka, J. (1966). Some Remarks on the Concepts of Nationalism and Internationalism. Historica, XIII: 209-216.

Korzeniewicz, R., \& Smith, W. (2004). Redes regionales y movimientos sociales transnacionales en patrones emergentes de colaboración y conflicto en las Américas. América Latina Hoy (36): 101-139.

Kriesberg, L. (1997). Social Movements and Global Transformation. En: J. Smith, C. Chatfield \& R. Pagnucco (Eds.), Transnational Social Movements and Global Politics: Solidarity beyond the States (Vol. Syracuse University Press: 3-18). Syracuse.

Lacan, J. (1973). Le séminaire. Livre XI : les quatre concepts fondamentaux de la psychanalyse, 1964 (Vol. XI). Paris: Éditions du Seuil.

Mann, M. (2002). ¿Ha terminado la globalización con el imparable ascenso del Estado nacional? [Has Globalization Ended the rise of the nation-state?]. Zona Abierta (92/93): 175-211.

Marx, K. (1985). El Capital. Crítica de la economía política ( Scaron, trad. 12 ed. Vol. I/2). México D. F.: Siglo veintiuno editores.

Marx, K. (1987). El Capital. Crítica de la economía política ( Scaron, trad. 16 ed. Vol. I/1). México D. F.: Siglo Veintiuno Editores.

McCarthy, J. D. (1997). The Globalization of Social Movement Theory. En: J. Smith, C. Chatfield \& R. Pagnucco (Eds.), Transnational Social Movements and Global Politics: Solidarity beyond the States (Vol. Syracuse University Press: 243259). Syracuse.

McMichael, P. (2004). Development and Social Change: A Global Perspective (3rd ed.). Thousand Oaks Pine Forges Press.

McMichael, P. (2008). Peasants Make Their Own History, But Not Just as They Please... En: Journal of Agrarian Change, 8 (23): $205-228$

Munck, R. (2004). Globalization and Social Exclusion: A Transformationalist Perspective. Bloomfield: Kumarian Press.

Munck, R. (2007). Globalization and Contestation: The New Great Counter-Movement. London; New York: Routledge.

Múnera Ruiz, L. (1998). Rupturas y continuidades: Poder y movimiento popular en Colombia 1968-1988. Santa Fe de Bogotá: CEREC; Universidad Nacional de Colombia.

O'Brien, R., Goetz, A. , Scholte, J. \& Williams, M. (2000). Contesting Global Governance: Multilateral Economic Institutions and Global Social Movements. Cambridge, UK: Cambridge University Press.

Pinto, M., \& Gálvez, C. (1996). Análisis documental de contenido. Procesamiento de la información. Madrid: Ed. Síntesis.

Podobnik, B. (2005). Resistance to Globalization: Cycles and Trends in the Global Protest Movement. En: B. Podobnik \& T. Reifer (Eds.), Transforming Globalization: Challenges and Opportunities in the Post 9/11 Era (51-68). Leiden: Brill.

Polanyi, K. (2001). The Great Transformation: The Political and Economic Origins of Our Time. Boston: Beacon Press.

Poulantzas, N. (1971). Pouvoir politique et classes sociales. Paris: François Maspero.
Poulantzas, N. (1986). Estado, poder y socialismo: Siglo Veintiuno Editores.

Reitan, R. (2007). Global Activism. London; New York: Routledge.

Santos, B. (2003). La caída del Angelus Novus: Ensayos para una teoría social y una nueva práctica política. Bogotá: ILSA.

Sassen, S. (1996). Losing Control?: Sovereignity in an Age of Globalization. New York: Columbia University Press.

Schmitt, C. (1990). Légalité et légitimité Du politique « légalité et légitimité » et autres essais (39-79). Paris: Pardès.

Scholte, J. (2005). Globalization: A Critical Introduction (2nd ed.). New York: Palgrave Macmillan.

Smith, J. (1997). Social Movements and World Politics. En: J. Smith, C. Chatfield \& R. Pagnucco (Eds.), Transnational Social Movements and Global Politics: Solidarity beyond the States (Vol. Syracuse University Press: 59-77). Syracuse.

Smith, J. (2002). Globalizing Resistance: the Battle of Seattle and the Future of Social Movements. En: J. Smith \& H. Johnston (Eds.), Globalization and Resistance: Transnational Dimensions of Social Movements (207-227). Lanham, Maryland: Rowman \& Littlefield.

Smith, J. (2005). Exploring Connections between Global Integration and Political Mobilization. En: B. Podobnik \& T. Reifer (Eds.), Transforming Globalization: Challenges and Opportunities in the Post 9/11 Era (29-49). Leiden: Brill.

Sommier, I. (2003). Le renouveau des mouvements contestataires : à l'heure de la mondialisation. Paris: Flammarion.

Tarrow, S. (1994). Power in Movement: Social Movements, Collective Action and Politics. Cambridge: Cambridge University Press.

Tarrow, S. (1998). Fishnets, Internets, and Catnets: Globalization and Transnational Collective Action. En M. Hanagan, L. Moch \& W. T. Brake (Eds.), Challenging Authority: the Historical Study of Contentious Politics (Vol. 7: 228-244). Minneapolis: University of Minnesota Press.

Tarrow, S. (2005). New Transnational Activism. Cambridge: Cambridge University Press.

van der Linden, M. (2003). Transnational Labor History: Explorations. Hants: Ashgate.

van Dijk, T. (1996). Análisis del discuso ideológico. Versión(6): 15-43.

Webster, N. (2004). Understanding the Evolving Diversities and Originalities in Rural Social Movements in the Age of Globalization. Geneva: Civil Society and Social Movements Programme, United Nations Research Institute for Social Development.

Wood, L. J. (2005). Taking to the Streets against Neoliberalism: Global Days of Action and Other Strategies. En B. Podobnik \& T. Reifer (Eds.), Transforming Globalization: Challenges and Opportunities in the Post 9/11 Era (69-81). Leiden: Brill.

World Commission on the Social Dimension of Globalization. (2004). A Fair Globalization: Creating Opportunities for All Retrieved Abril 12, 2010, from http://www.ilo.org/public/english/wcsdg/docs/report.pdf

Zamosc, L. (2001). Peasant Struggles in the 1970s in Colombia. En: S. Eckstein (Ed.), Power and Popular Protest: Latin American Social Movements (2nd ed., 102-131). Berkeley; Los Angeles; London: University of California Press.

Žižek, S. (2003). Da Capo senza Fine (C. Sardoy \& G. Homs, trad.) Contingencia, hegemonía, universalidad (215-262). Buenos Aires: Fondo de Cultura Económica. 